$\underline{\underline{p}}$

\title{
Automated segmentation of knee thermal imaging and X-ray in evaluation of rheumatoid arthritis
}

\author{
U. Snekhalatha*, T. Rajalakshmi, M. Gobikrishnan \\ Department of Bio medical Engineering, SRM Institute of science and Technology Chennai, Tamil Nadu, India \\ *Corresponding author E-mail: sneha_samuma@yahoo.co.in,
}

\begin{abstract}
Rheumatoid arthritis (RA) is a long lasting autoimmune disorder that affects the multiple joints of human body. The aim and objective of the study was i) to implement the automated segmentation of knee x-ray image and thermal image using fuzzy c means and canny edge detection algorithm. ii) To compare both the imaging modalities by means of feature extraction and correlate with the biochemical method as standard. Fifteen subjects with RA in knee region and 15 healthy controls were included in this study. The segmentation of thermal images was performed using fuzzy c-means algorithm and x-ray segmentation was implemented using canny edge detection algorithm. The skin surface temperature were measured in the thermal image of knee region in both RA and control subjects. The features were extracted from the segmented region of the knee x-ray image. The automated segmentation implemented in thermal imaging provided better results compared to $\mathrm{x}$-ray image segmentation process. The thermal imaging feature and $\mathrm{x}$-ray imaging features correlated significantly with the standard parameters.
\end{abstract}

Keywords: Canny Edge Detection, Fuzzy C Means Algorithm, Rheumatoid Arthritis, Thermal Imaging Method, X-Ray,

\section{Introduction}

Rheumatoid arthritis (RA) is a chronic systemic illness and a kind of chronic disorder that primarily affects the finger joints, hand, wrist, knee, elbow, shoulders and feet. It typically results in warm, inflammation of joints, swelling, pain and morning stiffness. The vital sub organs of body such as lungs, heart and eyes are affected and cause morbidity and mortality. The main cause of RA is not found clearly, it may be due to involvement of genetic and environmental factors. [1]. In RA, white blood cells attacks the joint tissues and travel to the synovium and cause inflammation (synovitis), characterized by warmth, redness, swelling, and pain. Currently conventional laboratory tests and radiography are used as gold standard method for diagnosis of RA. Worldwide, the prevalence of RA is predicted to be in the range from $0.4 \%$ to $1.3 \%$ [2-3].The synovial membrane becomes swollen in the arthritic knee and in severe cases bone erosion occurs between the joints. Hence it is very much required to detect the arthritis at any early stage for proper control over the disease. The biochemical method and x-ray method have been considered as 'gold' standard method in the diagnosis of RA according to Indian Rheumatology Association (IRA) consensus report [4]. Although both the biochemical method and x-ray method are gold standard for diagnosis of RA, these method has the following shortcomings: i) The biochemical method may not give complete clinical information about the progression of the disease; ii) X-ray method could not able to detect early changes and signs of RA in the soft tissues and bone erosion. Hence new methods such as MRI, thermal imaging and ultrasound are required in the evaluation of RA.
$\mathrm{X}$-rays of the hand and knee region are generally performed in patients with multiple joints affected by RA. Though there may be no changes in the early stages of RA, the parameters such as bone erosions, joint space narrowing, joint space destruction and cartilage destruction was predominantly visualized in $\mathrm{x}$-ray imaging of RA [5]. Hence as the patients and controls are subjected to radiation in X-ray, a non invasive diagnostic approach of thermal imaging is used in diagnosis of RA at an early stage. [6, 7]. Since manual method subject to inter observer variations, the automated method of segmentation provides better results in both the imaging modalities.

\section{Literature Works}

Several researchers have proposed and utilized the thermal imaging modalities and $\mathrm{x}$-ray in hand and knee regions [8-11]. Subramanian et al performed the non invasive method of osteoarthritis diagnosis in digital $\mathrm{x}$-ray images of knee region. In their study, they extracted the haralick features using GLCM method and implemented classification of normal and abnormal osteoarthritis using support vector machine [12]. Shamir et al described a automated method of OA in knee region based on KL grading method. They classified the normal and OA images using feature extraction techniques. The features were extracted from the transformed image [13]. Mengko et al implemented the automated method for the detection of Knee OA by means of measurement of joint space narrowing [14].

Several authors have performed automated segmentation in knee region of RA and evaluated its performance based on feature extraction and classification [15-17]. Salisbury et al observed the increased temperature in the lateral part of the knee, particularly in 
patellae region of RA compared to healthy controls. They found that the abnormal joints have localized hot spot regions with negative temperature gradient whereas the normal joints do not have increased temperature or hot spot regions [18]. Glehr et al evaluated the correlation between the skin surface temperatures at knee joint with the anterior knee pain implantation of an artificial knee joint. They computed the difference in temperature $\Delta \mathrm{T}$ between the average temperature of two different regions in contra lateral knees using the semi-automated approach. They observed the significant correlation between the anterior knee pain and increased temperature at the patellar region of the knee [19].

The aim and objectives of the study were i) to implement automated segmentation of knee x-ray image and thermal image using fuzzy c means (FCM) and canny edge detection algorithm respectively. ii) To compare both the imaging modalities by means of feature extraction and correlate with the biochemical method as standard.

\section{Materials and Methods}

\subsection{Subjects}

The subjects with knee RA considered as patients and patients without any knee problem considered as control subjects. 15 subjects with RA in knee region and 15 subjects without RA were included in this study.

i.Inclusion criteria

The patient who satisfies the revised American college of Rheumatology ACR criteria and. Indian Rheumatology consensus report were included in the study. The disease duration should be less than one year. The patient had given the informed consent for their active participation in this study.

ii.Exclusion criteria

The patients should have no clinical evidence of knee involvement. Patients who have joint pain for reasons other than RA cannot be considered in this study. Patients who have recently suffered bruising or trauma of the affected joints is not eligible for this study. Patients with joint sepsis and other clinical unstable medical conditions can be precluded.

\subsection{X-ray and Thermal Image Acquisition}

The digitized X-Ray imaging was acquired in patient's knee region using the standard $\mathrm{x}$-ray machine. The thermal images of the knee were acquired a posterior view of standing position using thermal camera FLIR SC 305. The studied population were exposed their knee in a temperature controlled with the humidity of $20^{\circ} \mathrm{C}$ for a period of 20 minutes prior to image acquisition. The patients were informed about the imaging procedure and the background region was covered with black colour sheet to acquire good thermal image in order to prevent the undesired artefacts in the image. The thermal imaging camera was positioned a head of the subjects with a gap of 1meter and the images were captured and analyzed using the FLIR software. The thermal images predicted hot spot regions for diseased patient, whereas no hot spot regions were found for normal.

\subsection{Knee Thermal Image Segmentation}

FCM algorithm is used in this study for image segmentation of thermal images. The FCM was popularized by ruspini and later continued by Dunn and Bezdek and has been widely used in cluster analysis, pattern recognition and image processing [20]. This method based on segmenting the abnormal region of interest by means of clustering techniques. FCM is a clustering technique in which the input features are grouped into $\mathrm{n}$ clusters with every feature in the dataset belonging to every cluster to a certain degree. The algorithm implemented using FCM algorithm was described as given below:

Step 1: Read and display the input image

Step 2: convert to rgb image plane to gray scale

Step 3: Compute the features from the image and select the number of clusters as $\mathrm{N}=3$

Step 3: Determine the cluster centre points using centroid formula Step 4: compute the average values of the cluster data points

Step 7: Cluster the image by calculating the distance between the cluster centre and the other regions in the image

Step8: The segmented output image is obtained and the segmented part superimposed on the original gray scale image.

\subsection{Knee Radiograph Segmentation}

The knee radiograph segmentation is carried out using canny edge detection technique. Canny edge detection detects the edges of the region of interest. This method is the most commonly used for edge detection and image segmentation. The Canny edge detection method is deliberate as one of the excellent edge detection algorithm for segmentation of knee $x$-ray image .Hence it segments the true edges or intensity discontinuities and provides good noise immunity while preserving a minimum error.

The $\mathrm{x}$-ray image segmented using canny edge detection method was given below:

Step 1: Read and display the knee $\mathrm{x}$-ray image

Step 2: Apply median Filter to remove the background noise.

Step 3: convert the filtered image into the binary image

Step 4: Adjust the contrast of the image using adaptive histogram equalization technique

Step 5: Apply canny edge detection algorithm to the contrast adjusted image.

i. The Gaussian filter is applied to smoothen the image.

ii. The intensity of gradient image is obtained

iii. Non maxima suppression and double thresholding is applied

iv. Suppress the weak edges and enhance the strong edges by hysteresis

v. The segmented output image is obtained.

Step 6: The region contours superimposed on the segmented image.

\subsection{Statistical Analysis}

The datas were analyzed using SPSS software version 19.0 (SPSS Inc., Chicago, IL, USA). The Pearson correlation was obtained for the skin temperature parameter and $\mathrm{x}$-ray feature parameter with the standard parameters.

\section{Results}

\subsection{Thermal Image Segmentation}

Thermal Image segmentation was carried out for fifteen knee thermal images of RA Patients and 15 control subjects. The abnormal regions are segmented in the patient's thermal image, whereas there is no abnormality found in control subjects. In Figure 1(a) is the input image of the patient thermal image. (b) the segmented abnormal regions indicated as black spot in the image (c) segmented output image superimposed on gray thermal image. (d) input image of healthy control thermal image. (e) depicts the there was no hotspot region so the segmented image shown as blank but a samll spot is identified due to noise effect during the acquistion process. (f) indicates the segmented image superimposed on gray scale image for healthy individuals. 

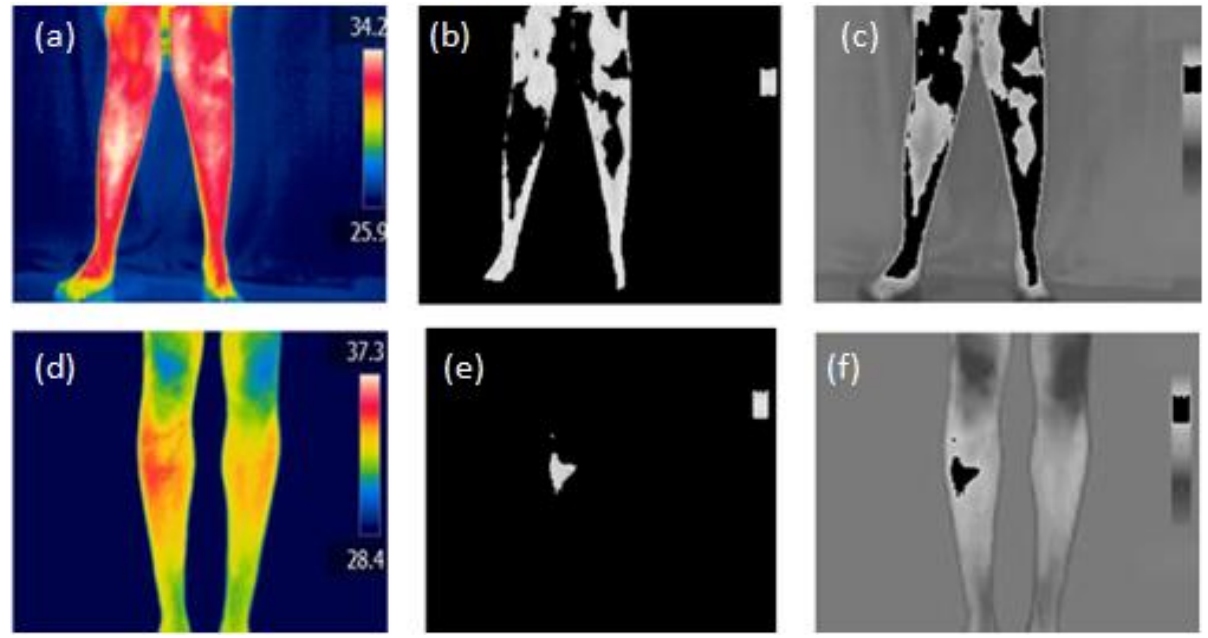

Fig.1: Image Segmentation of thermal images of RA patient and normal in knee region

\subsection{Knee Radiograph Analysis}

The knee x-ray image was segmented for fifteen RA patients and 15 control subjects. Figure 2 (a) indicates the input image of the healthy control and (b)binary image after thresholding (c) adaptive histogram equalized image (d) segmented iamge using canny edge detector. Figure 3(a) indicates the input image of the RA Patient (b) binary image after thresholding (c) adaptive histogram equalized image (d) segmented image using canny edge detection algorithm.
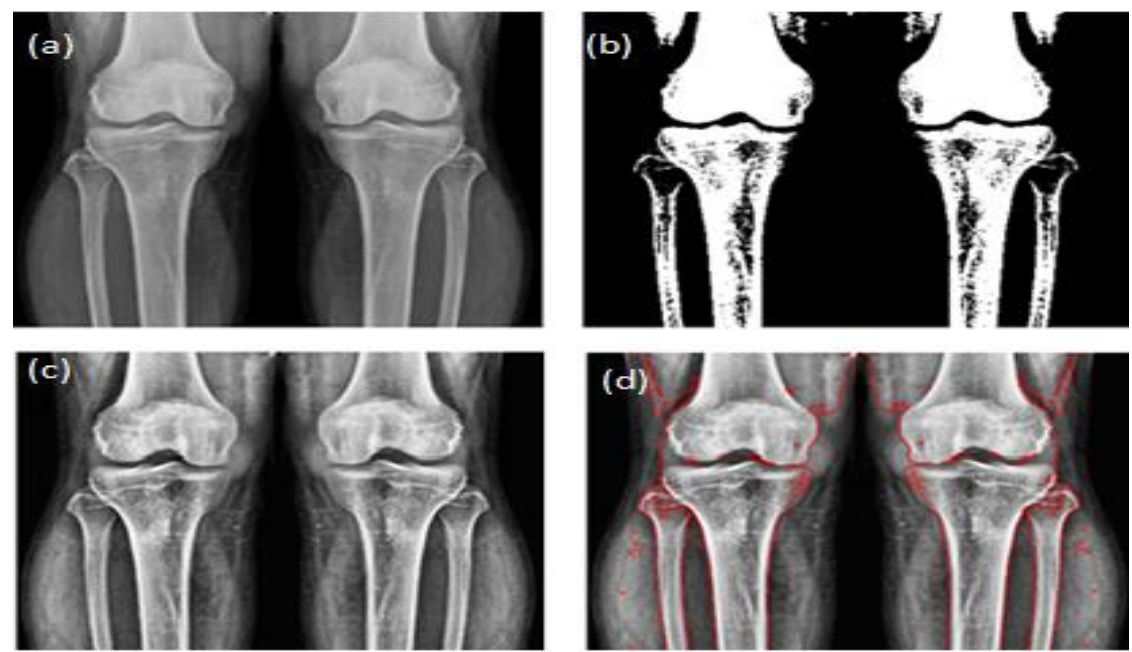

Fig.2: X-ray Image segmentation of normal subject
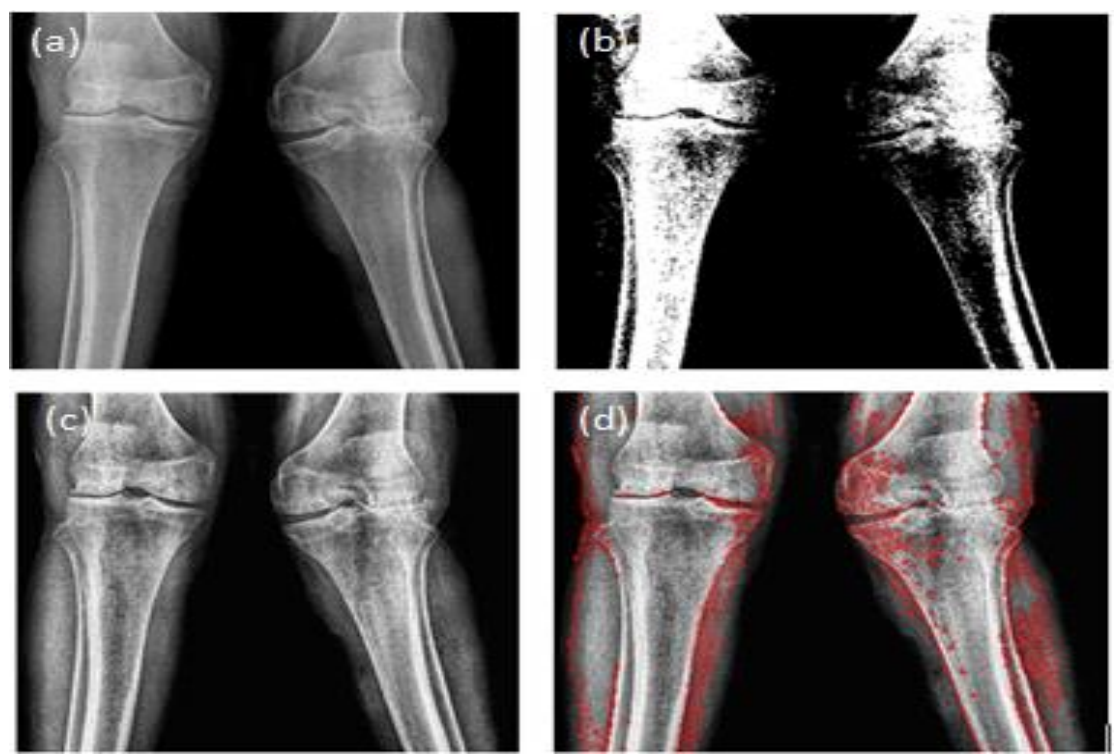

Fig.3: X-ray Image segmentation of RA patient 
Table 1: Correlation study between thermography and radiography with standard biochemical parameters

\begin{tabular}{|c|c|c|c|c|c|}
\hline \multirow{3}{*}{ S.No } & \multirow{3}{*}{ Parameters } & \multirow{2}{*}{\multicolumn{2}{|c|}{ Knee average Skin temperature }} & \multicolumn{2}{|l|}{$x$-ray image feature } \\
\hline & & & & Mean & \multirow{2}{*}{$\begin{array}{l}\text { entropy } \\
\text { Statisitcal signifcance }\end{array}$} \\
\hline & & Correlation cofficient ( $\mathbf{R})$ & $\begin{array}{l}\text { Statistical } \\
\text { significance }\end{array}$ & $\begin{array}{l}\text { Correlation cofficient } \\
\text { (R) }\end{array}$ & \\
\hline 1 & ESR & $0.775 * *$ & 0.001 & $0.679 * *$ & 0.001 \\
\hline 2 & Pain & $0.739 * *$ & $0 . .001$ & $0.646^{* *}$ & 0.003 \\
\hline 3 & Disability & $0.740 * *$ & 0.001 & $0.681 * *$ & 0.001 \\
\hline 4 & Health Status & $0.391 *$ & 0.005 & $0.616^{* *}$ & 0.002 \\
\hline
\end{tabular}

$* \mathrm{p}<0.05, * * \mathrm{p}<0.01$

The thermography correlation was made between biochemical tests, patient questionnaire score and knee average skin temperature for the total population studied $(\mathrm{N}=30)$. Among all the parameters, the biochemical parameter correlated significantly with the thermal imaging feature and $\mathrm{x}$-ray image features. The $\mathrm{x}$ ray image features were correlated with the standard parameters and found highly significant correlation in disability parameter compared to other parameters.

\section{Discussion}

In this study two different imaging modalities such as thermography and radiography was compared to biochemical method as standard. Snehalatha et al carried out the study on diagnosis of RA in hand region using thermal imaging method. In that study they compared the performance of EM algorithm and FCM algorithm in hand image segmentation. They found that FCM provided better results comparing to EM algorithm [21]. In this study automated segmentation of hotspot region in knee thermography was performed using FCM algorithm and obtained the promising results. Jacob et al performed manual and automated segmentation of knee region in $\mathrm{x}$-ray imaging using Otsu thresholding method. They obtained better segmentation result in segmenting the tibia bone region [22]. In this study the canny edgedetection clearly segmented the edges of the bone region in $\mathrm{x}-$ ray image. Subramoniam et al classified the arthritis in knee $\mathrm{x}$-ray images using K-Nearest Neighbour (KNN) and bayesian classifiers based on feature extraction from local binary pattern [23]. They used Euclidean distance between vectors for KNN and kernel distribution for bayesian classifier to extract features. In this study, the feature extracted parameter of thermography and radiography correlated with standard biochemical parameters and obtained significant results. The limitation of this study is need to obtain better segmentation results in $\mathrm{x}$-ray imaging by applying some advanced segmentation algorithm and validate the study by including more number of datas.

\section{Conclusion}

In conclusion, totally thirty thermal images and $30 \mathrm{x}$-ray images was used for automated segmentation process for the evaluating the RA using both $x$-ray and thermal imaging modalities. The automated segmentation implemented in thermal imaging provided better results compared to $\mathrm{x}$-ray image segmentation process. The thermal imaging feature and $\mathrm{x}$-ray imaging features correlated significantly with the standard parameters.

\section{Acknowledgements}

The authors would like to provide their sincere gratitude to SRM hospital and research centre for granting the infrastructure facilities to acquire the data from the patients.

\section{References}

[1] J.Jirholt, A.B.Lindquist, R.Holmdahl "The genetics of rheumatoid arthritis and the need for animal models to find and understand the underlying genes". Arthritis Res Vol 3. pp. 87-97, 2001.

[2] A.J. Silman, M.C.Hochberg. "Epidemiology of the rheumatic diseases". 2nd edition: Oxford University Press; 2001.

[3] J.J. Sacks, Y.H. Luo, C.G. Helmick. "Prevalence of specific types of arthritis and other rheumatic conditions in the ambulatory health care system in the United States 20012005”. Arthritis Care Res, Vol 62. pp.460-464, 2010

[4] R. Mishra, B.L.Sharma, R.Gupta, S.Pandya, S.Agarwal, P.Agarwal, S.Grover, P.Sarma, K.Wanjam, "Indian Rheumatology Association consensus statement on the management of adults with rheumatoid arthritis". Indian Journal of Rheumatology, Vol 3. pp.S1-S16, 2008

[5] J.Oliver, Sommer, A.Kladosek, V.Weiler, H.Czembirek, M.Boeck, M.Stiskal, "Rheumatoid arthritis: A Practical guide to state-of -the art imaging, image interpretation, and clinical implications", RSNA Radiographics Vol.25. 2005

[6] doi:http://dx.doi.org/10.1148/rg.252045111

[7] R. Lasanen, E. Piippo-Savolainen, T.Remes-Pakarinen, L.Kroger, A.Heikkila, P.Julkunen, J.Karhu, J.Toyras "Thermal imaging in screening of joint inflammation and rheumatoid arthritis in children". Physiol Meas, Vol 36. pp 273-82, 2015

[8] A.E. Denoble, N.Hall, C.F.Pieper, V.B.Kraus, "Patellar skin surface temperature by thermograhy reflects knee osteoarthritis severity". Clin Med Insights Arthritis Musculoskelet Disord, Vol 3 pp.69-75, 2010

[9] U.Snekhalatha, M.Anburajan, V.Sowmiya, B.Venkatraman, M.Menaga, "Automated hand thermal image segmentation and feature extraction in evaluation of rheumatoid arthritis" Proceedings of the Institution of Mechanical Engineers, Part $\mathrm{H}$ : Journal of Engineering in Medicine, Vol 229. pp.319-331, 2015

[10] A.B.Suma, U.Snekhalatha, T.Rajalakshmi, "Automated thermal image segmentation of knee rheumatoid arthritis", Proceedings of International conference on communication and signal processing, pp 535-539, 2016

[11] A.B.Suma, U.Snekhalatha, T.Rajalakshmi, "Evaluation of Rheumatoid arthritis in thermography and color Doppler ultrasound". IJCTA, Vol 9. pp 443-457, 2016

[12] M. Frize, J.Karsh, C.Herry, C.Adea, I.Aleem, P. Payeur, "Preliminary results of severity of illness measures of rheumatoid arthritis using infrared imaging," International workshop on medical measurements and applications., pp. 187-192, 2009, doi 10.1109/MEMEA.2009.5167981

[13] Subramoniam M, Barani S, Rajini V. A non invasive computer aided diagnosis of osteoarthritis from digital x-ray images Biomedical Research Vol 26, pp 721-729, 2015

[14] Shamir L, Ling SM, Scott WW, Bos A, Orlov N, Macura T, Eckley M, Ferrucci L, Goldberg G. Knee x-ray image analysis method for automated detection of osteoarthritis. Vol 56, pp $407-$ $415,2009$. 
[15] Mengko TL, Wachjudi RG, Suksmono AB, Danudirdjo D. Automated detection of unimpaired joint space for knee osteoarthritis assessment. Proc 7th Intl Workshop on Enterprise Networking and Computing in Healthcare Industry. pp400-403, 2005.

[16] Skala K, Lipic T, Sovic I, Gjenero J, Grubisic I, 4D thermal imaging for medical applications, Periodicum Biologorum, Vol 113, 407-416, 2011.

[17] Ju X, Nebel C, Siebert JP 3D thermography imaging standardization technique for inflammation diagnosis, Proc. of SPIE pp 5640-5646, 2004

[18] Spalding S J, Kwoh CK, Boudreau R, Enama J, Lunich J, Huber $\mathrm{D}$, Denes L, Hirsch $\mathrm{R}$ Three dimensional and thermal surface imaging produces reliable measures of joint shape and temperature: a potential tool for quantifying arthritis Arthritis Research \& Therapy 10: R10, 2008.

[19] Salisbury RS, Parr G, De Silva M et al. Heat distribution over normal and abnormal joints: thermal pattern and quantification. Ann Rheum Dis vol 42, pp 494-499,1983.

[20] Glehr M, StiborA, Sadoghi P, Schuster C, Quehenberger F, Gruber G, Leithner A, Windhager R. Thermal imaging as a non invasive tool for anterior knee pain following implantation of artificial knee joints International journal of Thermodynamics Vol 14, pp 71-78, 2011

[21] Y. Lu, T. Ma, C. Yin, X. Xie, W. Tian, S. Zhong, "Implementation of the Fuzzy C-Means Clustering Algorithm in Meteorological Data", International Journal of Database Theory and Application, Vol 6. pp. 1-18, 2013

[22] U.Snekhalatha, M.Anburajan, Therace Teena, B.Venkatraman, M.Menaka, Baldev Raj, "Thermal Image Analysis and segmentation of hand in Evaluation of Rheumatoid Arthritis". Proceedings of IEEE International conference on computer communications and informatics 2011; DOI: 10.1109/ICCCI.2012.6158784

[23] N.E. Jacob, M.V. Wyawahare, "Tibia Bone Segmentation in Xray Images A Comparative Analysis". International Journal of Computer Applications, Vol 76. 2013, DOI: 10.5120/13277-0873

[24] M. Subramoniam, V.Rajini, "Statistical Feature Based Classification of Arthritis in Knee X-Ray Images Using Local Binary Pattern". International conference on circuits, power and computing technologies, pp 873-875, 2013

[25] Harikishore Kakarla, Madhavi Latha M and Habibulla Khan, "Transition Optimization in Fault Free Memory Application Using Bus-Align Mode", European Journal of Scientific Research, Vol.112, No.2, pp.237-245, ISSN: 1450216x135/1450-202x, October 2013.

[26] T. Padmapriya and V. Saminadan, "Improving Throughput for Downlink Multi user MIMO-LTE Advanced Networks using SINR approximation and Hierarchical CSI feedback", International Journal of Mobile Design Network and InnovationInderscience Publisher, ISSN : 1744-2850 vol. 6, no.1, pp. 14-23, May 2015.

[27] S.V.Manikanthan and K.srividhya "An Android based secure access control using ARM and cloud computing", Published in: Electronics and Communication Systems (ICECS), 2015 2nd International Conference on 26-27Feb. 2015,Publisher:IEEE,DOI:10.1109/ECS.2015.7124833. 